\title{
Diminishing Returns in the Treatment of Metastatic Colorectal Cancer: Are Patients Reaching a Survival Plateau
}

\author{
Riccardo Lencioni ${ }^{1}$, James Chen ${ }^{2}$ \\ ${ }^{1}$ Department of Hepatology and Liver Transplantation, Pisa University Hospital and School of Medicine, Pisa, Italy; ${ }^{2}$ Light Sciences \\ Oncology, Inc., Bellevue, Washington, USA. \\ Email: lencioni@med.unipi.it
}

Received October $11^{\text {th }}$, 2010; revised October 23 $3^{\text {rd }}$, 2010; accepted October $27^{\text {th }}, 2010$.

\begin{abstract}
Despite the evolution of treatment for metastatic colorectal cancer (MCRC) over the past decade, improvements in survival endpoints have appeared to reach a plateau. The addition of expensive targeted biologic agents to the therapeutic armamentarium against MCRC have not drastically increased survival, particularly in the realm of second and third line patients, as tumor resistance remains an issue. Original approaches to treating MCRC are needed in order to raise the apparent survival ceiling in these patients.
\end{abstract}

Keywords: Colorectal Cancer, Chemotherapy, Molecular Targeted Agents

Metastatic colorectal cancer (MCRC) is a deadly solid tumor, and its incidence is still increasing in parts of the world, notably Asia [1]. In the United States there are approximately 150,000 new cases diagnosed per year [2]. Worldwide, approximately 1.2 million cases occur each year [3].

Drug therapy for MCRC has undergone an evolution over the past decade-from 5-fluorouracil (5-FU)-based therapy, to combination and sequential therapies that also incorporate the chemotherapeutics irinotecan and oxaliplatin, to the recent addition of targeted agents. Currently, patients with a good performance status typically begin on oxaliplatin plus 5-FU/leucovorin (FOLFOX) or irinotecan plus 5-FU/leucovorin (FOLFIRI), with bevacizumab added to either regimen. Median survival time under the best circumstances is about 20 months, provided the patient can tolerate exposure to multiple lines of chemotherapy [3]. Efforts to improve overall survival have resulted in the development of a number of targeted agents used at various stages of therapy, including bevacizumab, cetuximab, and panitumumab. Bevacizumab, approved by the FDA in combination with irinotecan, fluorouracil, and leucovorin, primarily targets the vascular endothelial growth factor (VEGF) pathway, while cetuximab and panitumumab target the epidermal growth factor (EGF) pathway [4,5]. Despite the addition of new targeted agents to the chemotherapy backbone, the 5-year survival of patients with metastatic disease is only $8 \%$ [6].

What has targeted therapy added in terms of MCRC survival? Bevacizumab has been investigated in the first-line setting in randomized trials. In a large double-blinded first-line study, patients received XELOX or FOLFOX4, then either bevacizumab or placebo. The response rate and median overall survival time were not significantly different between the two groups [3]. Bevacizumab has not been tested in a randomized controlled first-line setting with FOLFIRI, and pooled studies involving bevacizumab have not conclusively demonstrated that its addition to standard front-line regimens increases survival beyond what is currently achieved with FOLFOX or FOLFIRI alone [2,7,8]. Pooled analysis of the impact of adding bevacizumab to chemotherapy in a recent Cochrane review revealed a median survival of 20.5 months, which approximates survival achieved with usage of the three chemotherapeutic agents alone $[9,10]$.

Cetuximab has been demonstrated to increase median overall survival by 1.5 months in the third-line setting, but no difference has been noted in the second- or first-line setting [2,3]. Panitumumab has received the most recent United States approval for MCRC after failure of first- and second-line therapy. In the pivotal trial, 
no difference in overall survival was noted when compared to best supportive care alone [3].

Solid trial data and subsequent clinical experience support the use of the three main cytotoxic agents-irinotecan, oxaliplatin and the fluoropyrimidines - which by all accounts have increased survival in a meaningful manner [2, 3]. Studies involving cytostatic targeted agents have, on the other hand, demonstrated marginal incremental benefit, with significant added cost and emergence of new classes of side effects [2].

Arguably, a survival ceiling is being reached for most patients with MCRC. Ongoing investigations of the cytostatic drugs erlotinib, gefitinib, sunitinib, vatalanib, and other antiangiogenesis inhibitors are unlikely to provide further major survival improvements in patients with advanced CRC. Further, the PACCE and CAIRO2 trials, which combined VEGF and EGFR inhibitors with chemotherapy, demonstrated worse outcomes for the combinations of VEGF with EGFR inhibitors [6]. Current approaches in development are encumbered by intrinsic or acquired tumor resistance coincident with dose-limiting toxicity and high cost, preventing truly meaningful improvements in longevity and affordability, with fewer drug-related side effects. New, very original approaches to MCRC beyond targeted therapy deserve increased attention and development efforts.

\section{Acknowledgements}

The authors take full responsibility for the scope, direction and content of the manuscript. They would like to thank Research Analysis Library for the assistance in the literature review and in the preparation of the draft manuscript. Editorial assistance was supported by Light Science Oncology, Inc.

\section{REFERENCES}

[1] J. J. Sung, J. Y. Lau, G. P. Young, Y. Sano, H. M. Chiu, J. S. Byeon, K. G. Yeoh, K. L. Goh, J. Sollano, R. Rerknimitr, T. Matsuda, K. C. Wu, S. Ng, S. Y. Leung, G.
Makharia, V. H. Chong, K. Y. Ho, D. Brooks, D. A. Lieberman and F. K. Chan, "Asia Pacific Consensus Recommendations for Colorectal Cancer Screening," Gut, Vol. 57, No. 8, 2008, pp. 1166-1176.

[2] S. K. Maithel and M. I. D'Angelica, “An Update on Randomized Clinical Trials in Advanced and Metastatic Colorectal Carcinoma," Surgical Oncology Clinics of North America, Vol. 19, No. 1, 2010, pp. 163-181.

[3] C. H. Kohne and H. J. Lenz, "Chemotherapy with Targeted Agents for the Treatment of Metastatic Colorectal Cancer,” Oncologist, Vol. 14, No. 5, 2009, pp. 478-488.

[4] M. H. Cohen, J. Gootenberg, P. Keegan and R. Pazdur, "FDA Drug Approval Summary: Bevacizumab Plus FOLFOX4 as Second-line Treatment of Colorectal Cancer,” Oncologist, Vol. 12, No. 3, 2007, pp. 356-361.

[5] H. Hurwitz, L. Fehrenbacher, W. Novotny, T. Cartwright, J. Hainsworth, W. Heim, J. Berlin, A. Baron, S. Griffing, E. Holmgren, N. Ferrara, G. Fyfe, B. Rogers, R. Ross and F. Kabbinavar, "Bevacizumab Plus Irinotecan, Fluorouracil, and Leucovorin for Metastatic Colorectal Cancer," New England Journal of Medicine, Vol. 350, No. 23, 2004, pp. 2335-2342.

[6] P. Comella, R. Casaretti, A. Avallone and L. Franco, "Optimizing the Management of Metastatic Colorectal Cancer,” Critical Reviews in Oncology/Hematology, Vol. 75, No. 1, 2010, pp. 15-26.

[7] F. Montagnani, C. Migali and G. Fiorentini, "Progression-free Survival in Bevacizumab-based First-line Treatment for Patients with Metastatic Colorectal Cancer: Is It a Really Good End Point?” Journal of Clinical Oncology, Vol. 27, No. 28, 2009, pp. e132-133.

[8] S. Welch, K. Spithoff, R. B. Rumble and J. Maroun, "Bevacizumab Combined with Chemotherapy for Patients with Advanced Colorectal Cancer: A Systematic Review," Annual Oncology, Vol. 21, No. 6, 2010, pp. $1152-1162$

[9] J. Capdevila, F. J. Ramos, T. Macarulla, E. Elez and J. Tabernero, "The Role of Salvage Treatment in Advanced Colorectal Cancer," Critical Reviews in Oncology/Hematology, Vol. 71, No. 1, 2009, pp. 53-61.

[10] A. D. Wagner, D. Arnold, A. A. Grothey, J. Haerting and S. Unverzagt, “Anti-angiogenic Therapies for Metastatic Colorectal Cancer”. Cochrane Database of Systematic Reviews, No. 3, 2009, pp. CD005392. 